\title{
Searching for weak or complex magnetic fields in polarized spectra of Rigel
}

\author{
Matthew Shultz ${ }^{1,2}$, Gregg A. Wade ${ }^{2}$, Coralie Neiner ${ }^{3}$, Nadine \\ Manset $^{4}$, Véronique Petit ${ }^{2,5}$, Jason H. Grunhut ${ }^{1,2}$, Edward Guinan ${ }^{6}$, \\ David A. Hanes ${ }^{1}$ and the MiMeS collaboration \\ ${ }^{1}$ Queen's University, Canada ${ }^{2}$ Royal Military College, Canada ${ }^{3}$ Paris-Meudon Observatory \\ ${ }^{4}$ Canada-France-Hawaii Telescope Corporation ${ }^{5}$ West Chester $\mathrm{U}^{6}$ Villanova U
}

\begin{abstract}
Seventy-eight high-resolution Stokes V, Q and U spectra of the B8Iae supergiant Rigel were obtained with the ESPaDOnS spectropolarimeter at CFHT and its clone NARVAL at TBL in the context of the Magnetism in Massive Stars (MiMeS) Large Program, in order to scrutinize this core-collapse supernova progenitor for evidence of weak and/or complex magnetic fields. In this paper we describe the reduction and analysis of the data, the constraints obtained on any photospheric magnetic field, and the variability of photospheric and wind lines.
\end{abstract}

Keywords. stars: magnetic fields, stars: variables: other, stars: activity, stars: winds, outflows

\section{Introduction: Physical Parameters and Observations}

Rigel: a blue supergiant, the closest and most readily studied Type II supernova progenitor, and a known $\alpha$ Cygni variable, is the subject of a global monitoring campaign known as the 'Rigel-thon', involving long-term spectroscopic monitoring, Microvariability and Oscillations in STars (MOST) space photometry, and spectropolarimetry.

Like most OB stars, Rigel ( $\beta$ Ori A) shows no sign of an easily detected magnetic field, however apparent brightness and sharp spectral lines make it practical to ask if the star possesses a weak or complex field geometry which might be revealed within a high resolution data set. Thus, over the epoch 09/2009-02/2010, 65 Stokes V (circular polarization) and 13 Stokes Q and U (linear polarization) spectra spanning $370-1000 \mathrm{~nm}$ with a mean resolving power $\mathrm{R} \sim 65000$ at $500 \mathrm{~nm}$, were taken with the ESPaDOnS spectropolarimeter at CFHT and its clone Narval at TBL. Integration times were typically of a few seconds duration. The densest spectropolarimetric sampling was concurrent with the collection of MOST data.

The physical radius was determined from the interferometric angular diameter, $\theta_{D}=2.76 \pm 0.01$ mas (Aufdenberg et al., 2008) together with a distance of $240 \pm 50 \mathrm{pc}$ calculated using the Hipparcos parallax of $4.22 \pm 0.81$ mas: thus $\mathrm{R}=70 \pm 14 \mathrm{R}_{\odot}$. The star appears to be a slow rotator, with $v \sin i=36 \pm 5 \mathrm{~km} / \mathrm{s}$ (Przybilla et al. 2006), giving an upper bound on the rotation period $\mathrm{P}_{\text {rot }}$ of $\sim 98 \mathrm{~d}$; calculation of the breakup velocity $(\sim 250 \mathrm{~km} / \mathrm{s})$ provides a lower limit of $\sim 14 \mathrm{~d}$.

\section{Results and Analysis}

Least Squares Deconvolution (LSD) was employed to extract high S/N ratio mean Stokes I, V, and diagnostic N profiles from the circular polarization spectra. The LSD line mask was cleaned to eliminate contamination from telluric, emission and Balmer lines, and to remove weak or apparently absent lines. Ultimately $\sim 90$ lines remained, and their weights were empirically adjusted to reflect observed line depths. The typical 
$\mathrm{S} / \mathrm{N}$ ratio in Stokes $\mathrm{V}$ mean profiles was $\sim 20,000$. No significant signal was detected in either Stokes V or diagnostic N. Each LSD profile was then analyzed to determine the longitudinal magnetic field $\mathrm{B}_{l}$. No significant longitudinal field was detected, with a median 1- $\sigma$ uncertainty in individual measurements of $13 \mathrm{G}$. The distribution of $\mathrm{B}_{l}$ values inferred from Stokes $\mathrm{V}$ is statistically identical to that inferred from diagnostic $\mathrm{N}$. The measured $\mathrm{B}_{l}$ was then compared to a grid of synthetic longitudinal field curves corresponding to dipoles with $0^{\circ} \leqslant \beta \leqslant 90^{\circ}, 0^{\circ} \leqslant i \leqslant 90^{\circ}$ (with the data folded according to the theoretical maximum $\mathrm{P}_{\text {rot }}=93 \mathrm{~d}$ at $\mathrm{i}=90^{\circ}$ and progressively shorter periods at smalled $\mathrm{i}$, with ten different phase offsets tested at each period), and polar field strengths $\mathrm{B}_{d}$ from 0 to $\sim 3 \mathrm{kG}$. For $\left(\mathrm{i}=90^{\circ}, \beta=90^{\circ}\right)$ the maximum $\mathrm{B}_{d}$ compatible with the data at 3- $\sigma$ confidence is $\sim 20 \mathrm{G}$, while $\mathrm{B}_{d}$ is constrained below $\sim 50 \mathrm{G}$ for intermediate values of $\mathrm{i}$ and $\beta$. Fields at this level, if present within the photosphere, remain capable of strongly influencing the wind (ud-Doula \& Owocki, 2002), with a wind magnetic confinement parameter $\eta_{*} \sim 2-90$, assuming $\dot{M} \sim 10^{-7}-10^{-6} \mathrm{M}_{\odot} / \mathrm{y}$ (Barlow \& Cohen 1977, Abbot et al. 1980, Puls et al. 2008) and $v_{\infty} \sim 400-600 \mathrm{~km} / \mathrm{s}$ (Bates et al. 1980).

Rigel is a long-known $\alpha$ Cygni variable (Sanford, 1947), with significant line profile variability (LPV) in $\mathrm{H} \alpha$ as well as various metal lines, which may be associated with any or all of: mass loss events, photospheric spots, corotating interacting structures, and/or g- or p-mode pulsations (Kaufer et al. 1996a, 1997). Distinct LPV is seen in $\mathrm{H} \alpha$ as compared to metal lines: $\mathrm{H} \alpha$ is in strong emission, variable over a broad velocity range and apparently aperiodic (consistent with earlier spectroscopic monitoring, Kaufer et al. 1996a, 1996b, Israelian et al. 1997); metal lines showed little apparent emission excess, but their variability was suggestive of periodic behaviour. Amongst the most complexly variable of the metal lines is the $\mathrm{O}$ triplet at $777 \mathrm{~nm}$.

\section{Conclusions \& Future Work}

No evidence of a magnetic field is obtained in 65 high precision Stokes V observations of Rigel. Significant variability is observed in numerous spectral lines, with some suggestion of periodicity on the order of $\sim 1$ month in metallic lines. Further modeling of Stokes $\mathrm{V}$ profiles must be performed to obtain quantitative constraints on various potential field topologies, e.g. the dynamo-generated field proposed by Cantiello et al. (2009); a more rigorous analysis of LPV may help to identify periodic behaviour.

\section{References}

Abbott, D. C., Bieging, J. H., Churchwell, E., \& Cassinelli, J. P. 1980, ApJ, 238, 196

Aufdenberg, J. P., Ludwig, H.-G., Kervella, P., Mérand, A. et al. 2008, in: A. Richichi, F. Delplancke, F. Paresce, \& A. Chelli (eds.), The Power of Optical/IR Interferometry: Recent Scientific Results and 2nd Generation, ESO Astrophysics Symposia, p. 71

Barlow, M. J. \& Cohen, M. 1977, ApJ, 213, 737

Bates, B., Giaretta, D. L., McCartney, D. J., McQuoid, J. A. et al. 1980, MNRAS, 190, 611

Cantiello, M., Langer, N., Brott, I., de Koter, A. et al. 2009, A\&A, 499, 279

Israelian, G., Chentsov, E., \& Musaev, F. 1997, MNRAS, 290, 521

Kaufer, A., Stahl, O., Wolf, B., Gaeng, T. et al. 1996a, A\&A, 305, 887

Kaufer, A., Stahl, O., Wolf, B., Gaeng, T. et al. 1996b, A\&A A, 314, 599

Kaufer, A., Stahl, O., Wolf, B., Fullerton, A. W. et al. 1997, A\& A, 320, 273

Puls, J., Vink, J. S., \& Najarro, F. 2008, A\& AR, 16, 209

Przybilla, N., Butler, K., Becker, S. R., \& Kudritzki, R. P. 2006, A\&A, 445, 1099

Sanford, R. F. 1947, ApJ, 105, 222

ud-Doula, A. \& Owocki, S. P. 2002, ApJ, 576, 413 